Supplementary Materials for

\title{
Osmium isotope and trace elements reveal melting of Chhota Shigri Glacier, western Himalaya, insensitive to anthropogenic emission residues
}

5 SARWAR NizaM ${ }^{1 *}$, INDra SEKHAR SEN ${ }^{1}$, TANUJ SHUKLA $^{1}$, DAVID SELBY $^{2,3}$

${ }^{1}$ Department of Earth Sciences, Indian Institute of Technology Kanpur, Kanpur, UP 208016, India.

${ }^{2}$ Department of Earth Sciences, University of Durham, Durham DH1 3LE, UK.

${ }^{3}$ State Key Laboratory of Geological Processes and Mineral Resources, School of Earth

10 Resources, China

*Correspondence to: sarwar@iitk.ac.in; isen@iitk.ac.in

\section{This PDF file includes:}

Figure S1-S4 and their captions. 


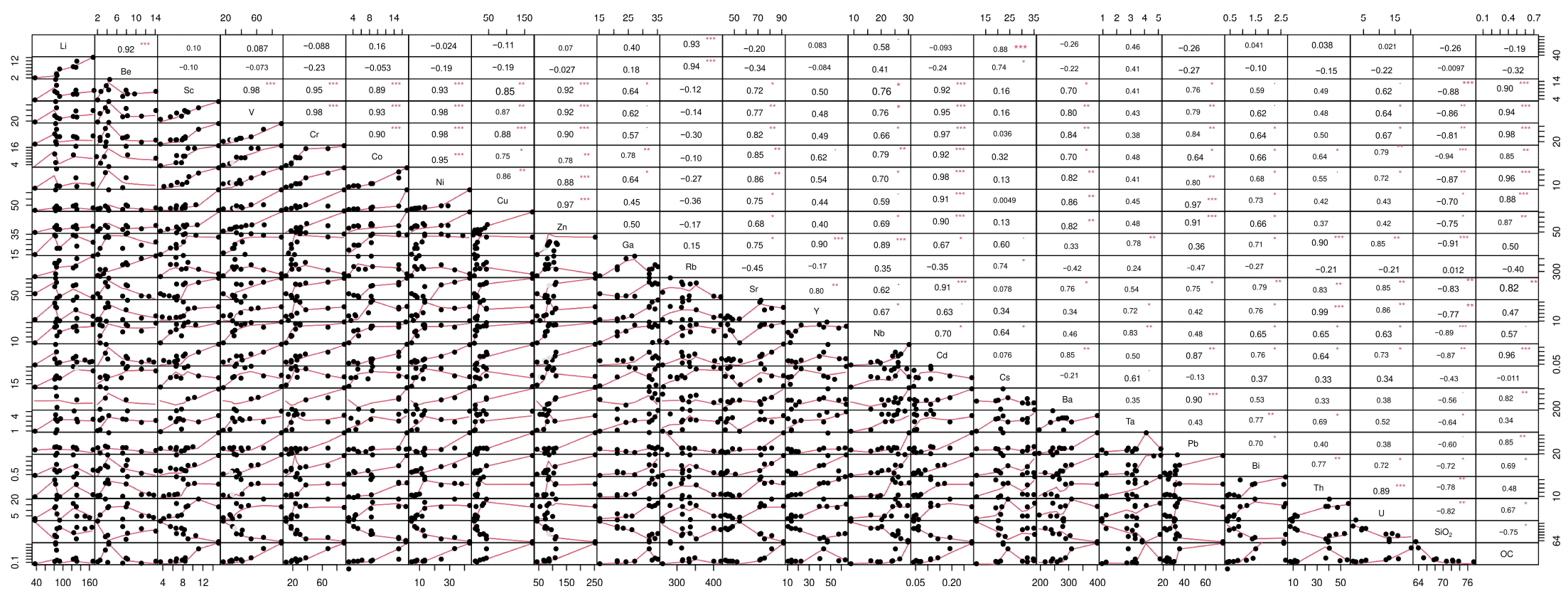

Figure S1. Distribution plot for each variable associated with trace metal and major oxides of moraine samples. On the bottom of the diagonal: the bivariate scatter plots with a fitted line are displayed. At the top of the diagonal: the value of the correlation plus the significance level as stars. Each significance level is associated to a symbol “***”, “**”, "**, ".", " “ and associated p-values are $0.001,0.01,0.05,0.1,1)$ respectively. 


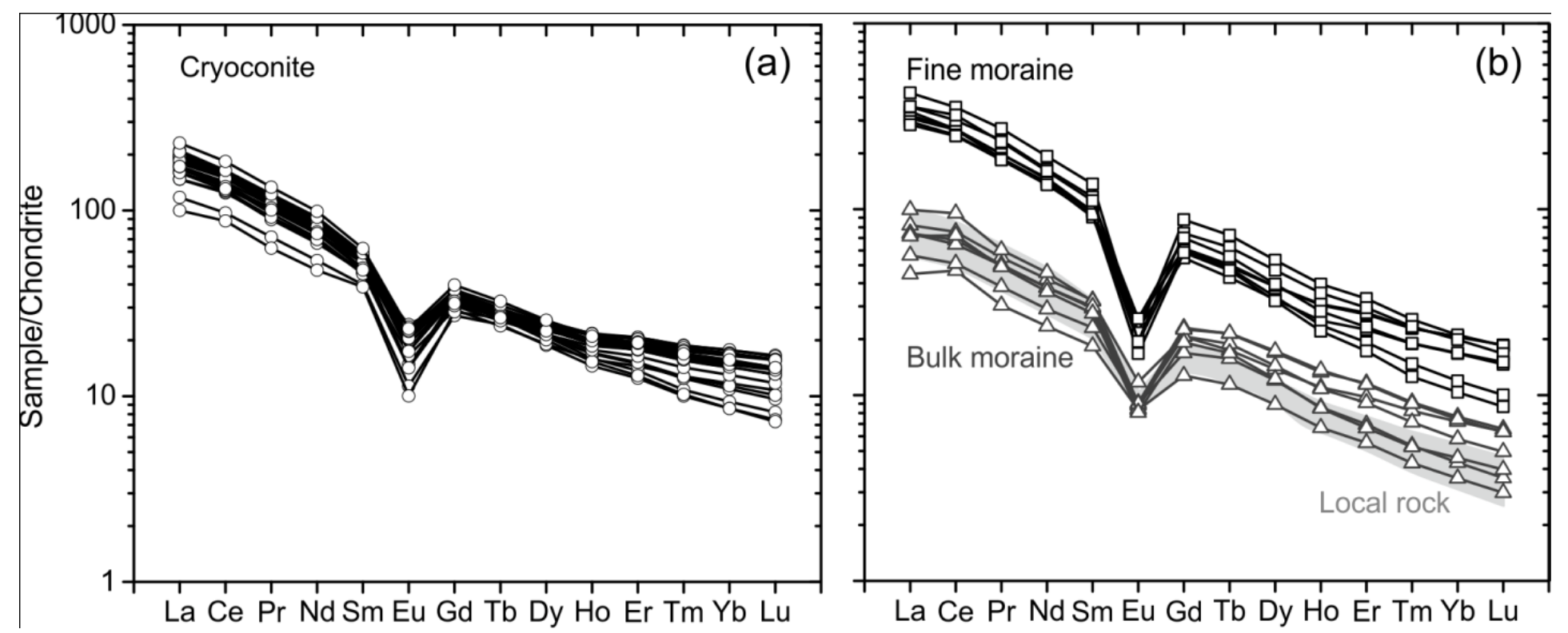

Figure S2. Chondrite normalized (McDonough and Sun, 1995) REE plots for individual cryoconite (a) and moraine debris (b) samples from ablation zone of CSG. 


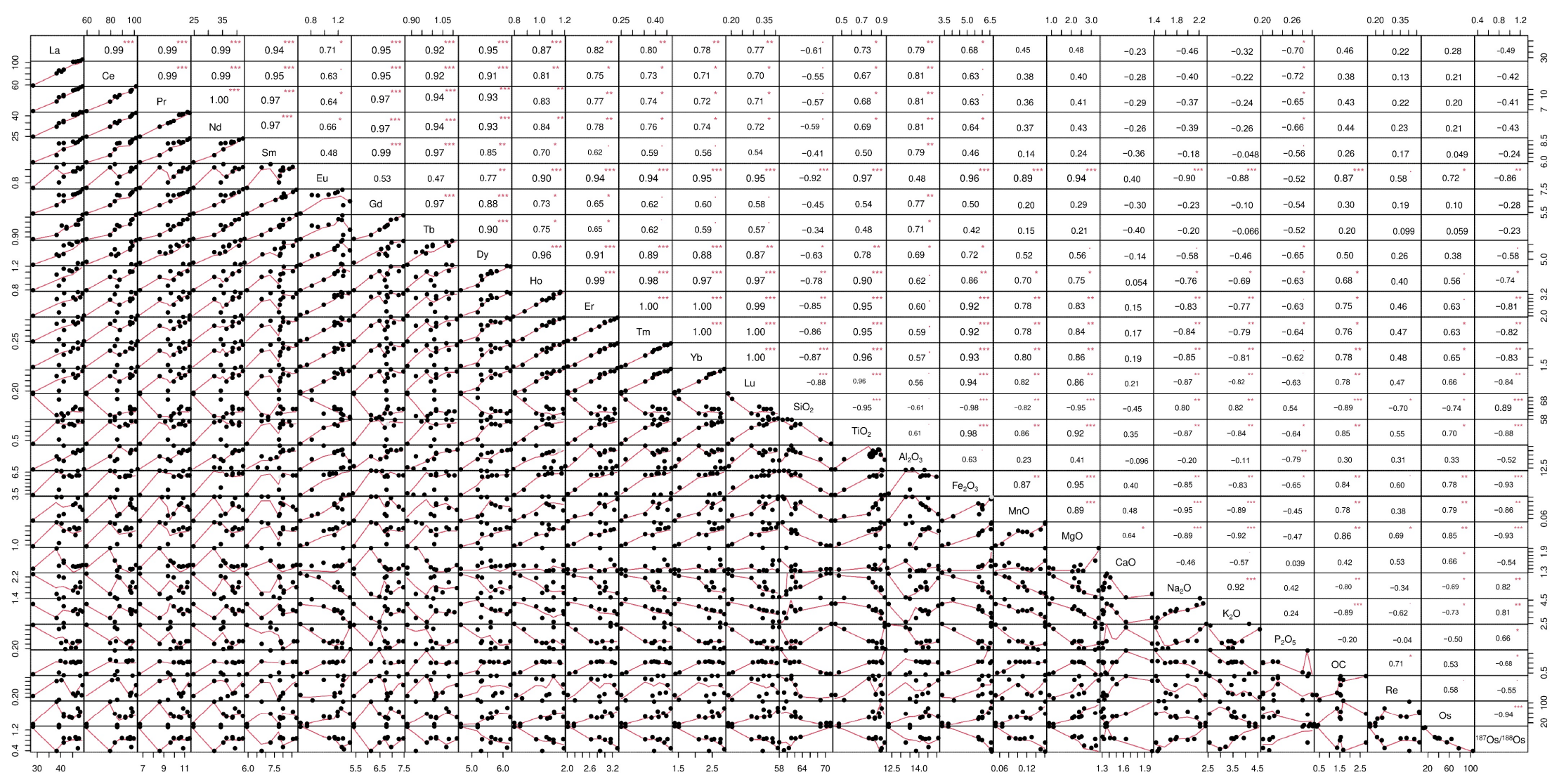

Figure S3. Distribution plot for each variable associated with REE, $\mathrm{SiO}_{2}$, TOC, Re-Os concentration and ${ }^{187} \mathrm{Os} /{ }^{188} \mathrm{Os}$ ratio of cryoconite sample. At the top of the diagonal: the value of the correlation plus the significance level as stars. Each significance level is associated to a symbol “***”, "***, “*”, ".”, " "and associated p-values are.001,.01,.05, .1, 1) respectively. 


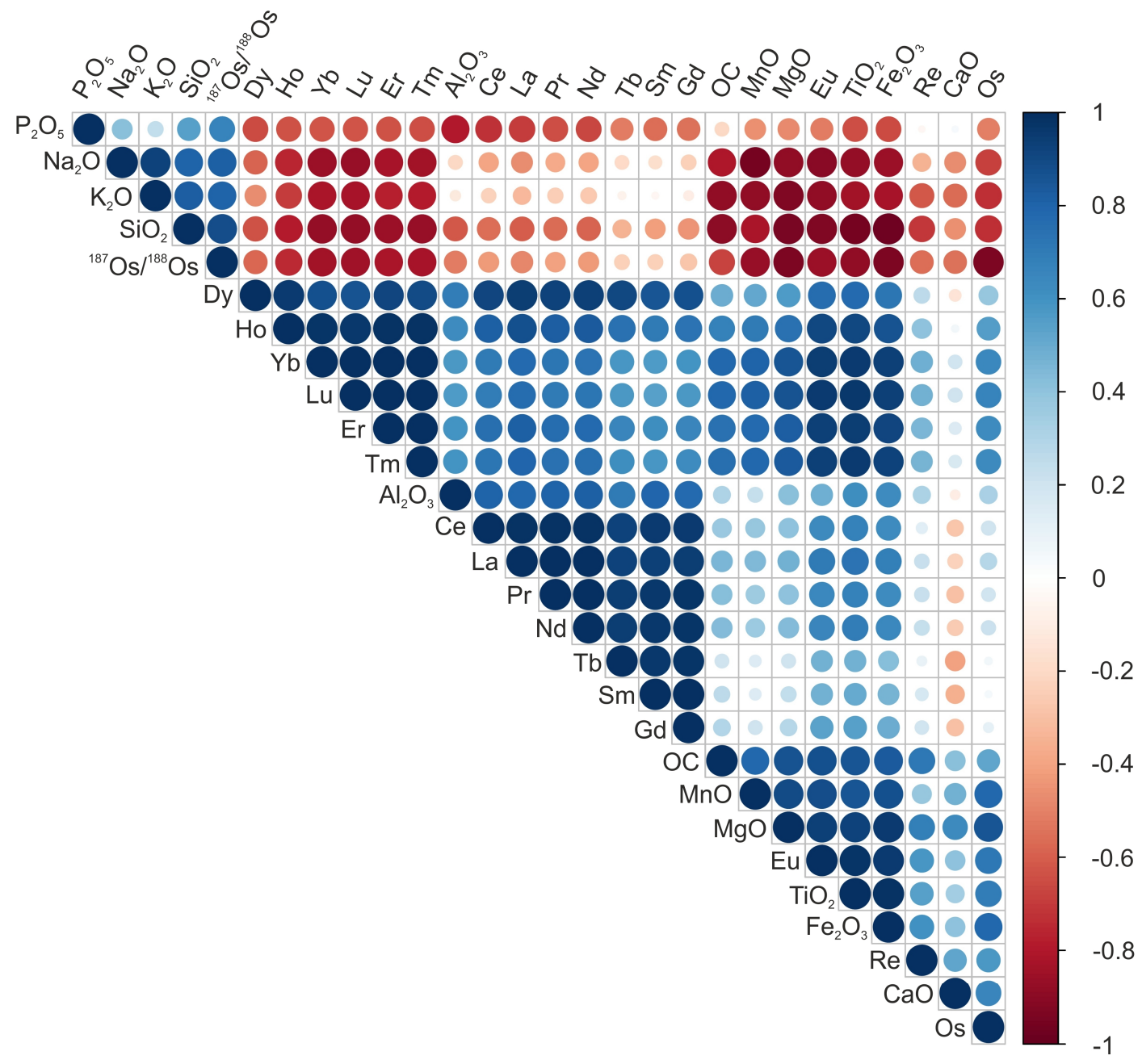

Figure S4. Correlogram showing Pearson correlation coefficient between REE and major oxide analyzed in cryoconite samples. Positive correlations are displayed in blue and negative correlations in red color. Color intensity and the size of the circle are proportional to the correlation coefficient. 